\title{
Sizing procedures for sun-tracking PV system with batteries
}

\author{
Ömer Nezih Gerek ${ }^{1, *}$, Ümmühan Başaran Filik ${ }^{1}$, and Tansu Filik ${ }^{1}$ \\ ${ }^{1}$ Anadolu University, Faculty of Engineering, Eskisehir, 26555, Turkey
}

\begin{abstract}
Deciding optimum number of PV panels, wind turbines and batteries (i.e. a complete renewable energy system) for minimum cost and complete energy balance is a challenging and interesting problem. In the literature, some rough data models or limited recorded data together with low resolution hourly averaged meteorological values are used to test the sizing strategies. In this study, active sun tracking and fixed PV solar power generation values of ready-to-serve commercial products are recorded throughout 2015-2016. Simultaneously several outdoor parameters (solar radiation, temperature, humidity, wind speed/direction, pressure) are recorded with high resolution. The hourly energy consumption values of a standard 4-person household, which is constructed in our campus in Eskisehir, Turkey, are also recorded for the same period. During sizing, novel parametric random process models for wind speed, temperature, solar radiation, energy demand and electricity generation curves are achieved and it is observed that these models provide sizing results with lower LLP through Monte Carlo experiments that consider average and minimum performance cases. Furthermore, another novel cost optimization strategy is adopted to show that solar tracking PV panels provide lower costs by enabling reduced number of installed batteries. Results are verified over real recorded data.
\end{abstract}

\section{Introduction}

The share of renewable energy generations from solar and wind inside the overall electricity generation is rapidly increasing. The most critical drawbacks of using these renewable energy sources can be listed as their unpredictable nature, dependence on seasonal changes, and the variations of solar and wind energy, which may not match with the time distribution of load demand. For a stand-alone renewable energy system (wind-photovoltaic-battery), in order to continuously satisfy energy demand with lowest investment costs, a thorough sizing methodology is necessary. Several mathematical approaches are proposed in the literature $[1-3,5,6]$, most of them being obtained under relatively low resolution (i.e. hourly) wind / solar radiation readings) data recording. For a valid and accurate renewable energy system sizing result, it is typically required to have at least one-year high resolution (dense) wind speed, temperature, solar radiation, and energy demand values. These values depend on several atmospheric events, as well as the physio-chemical characteristics of the energy harvesting machinery (i.e., PV panels and turbine engines). Nevertheless, the sizing methods normally stop the process after a fixed 
period of data recording and training. Then, the algorithms run a time series analysis to come up with an optimized sizing that would minimize loss of load probability (LLP) if the same days would have appeared in the future $[5,6]$. Unfortunately, the performance of a determined size may not show the same performance in the future conditions, even if the overall sun light and wind conditions do not statistically change. For a more reliable sizing result, methods that involve wide-sense models and forecasting of wind and solar radiation could be considered [2]. An analysis for a wind energy integrated systems was developed in [8] to form a production simulation tool. In general, linear regression and ARMA based methods are preferred for wind speed modelling [9], whereas solar energy models range from geographical calculations, to linear models, to artificial neural networks [10]. In a recent study, the solar model was even constructed as a surface in two dimensions, with axes being hours and days [11]. In that particular work, stochastic data generation functions were fed with transition models for wind speed and electricity demand, and the 2D modelling approach was adopted for solar radiation modelling.

In our experiments, active sun tracking and fixed PV solar power generation values of ready-to-serve commercial products are recorded throughout years 2015-2016 in Eskişehir (an inland place around $30-31^{\circ}$ north latitude). At the same period, several outdoor parameters (solar radiation, temperature, humidity, wind speed/direction, pressure) are recorded with high resolution. Finally, in order to perform the sizing analysis using the demand side, hourly energy consumption values of a standard 4-person household are recorded from a constructed household emulator in our university campus in Eskisehir, Turkey. Using the above-mentioned set-up, parametrized random process models are extracted for wind speed, temperature, solar radiation, energy demand and electricity generation. Such modelling enables us to simulate the stand-alone renewable energy system's performance over several years. The Monte Carlo experimentation approach [7] that is used for modelling is explained in Sec. 2.2. By using these random process models to generate renewable data, it is possible to test the sizing results both in the average and in the worst-case sense. Finally, using a new sizing and cost analysis approach [3], a comparison between the size obtained from a fixed solar panel and an active solar tracking panel (under the same solar conditions) is made. The new model approaches the sizing problem with a combination of source cost and its corresponding LLP cost [3]. It is observed that the overall battery requirements for an active solar tracking system is sufficiently lower than that of a fixed panel system, making the cost of the system smaller, despite the initial higher cost of the tracker system.

\section{Data acquisition and modelling}

In order to come up with exact simultaneously time stamped data of solar radiation, wind speed, energy from PV, energy from wind, temperature, and actual energy demand, rigorous data acquisitions are achieved from a controlled experimental setup as shown in Figure 1. This experimental setup is inside the Anadolu University 2 Eylul Campus in Eskisehir region. The described data are logged in hard drives and in cloud based systems. The solar part of the produced energy was from (i) a single-axis active sun tracking PV panels, (ii) fixed PV panels, and iii) roof-mounted panels. These are accompanied by wind turbines. All of the utilized machinery were ready-to-serve commercial products, including the acquisition and recording systems. The energy demand was emulated using an actual construction (inside the same campus) which hosts a standard 4-person household with all average electrical consuming electronics and lighting products. The electrical machineries (such as refrigerator, lights, etc.) were run according to reported average usage values in Turkey. Detailed technical specifications of the system can be reached from [4]. 


\subsection{Data sets}

Portions of the collected 20 seconds interval wind speed, temperature and solar radiation and pressure values are shown in Figure 2(a), (b), (c), and (d), respectively. Neither the presented wind speed, nor the solar radiation values correspond to the generated electrical energy. The energy conversion result is illustrated in Figure 3.

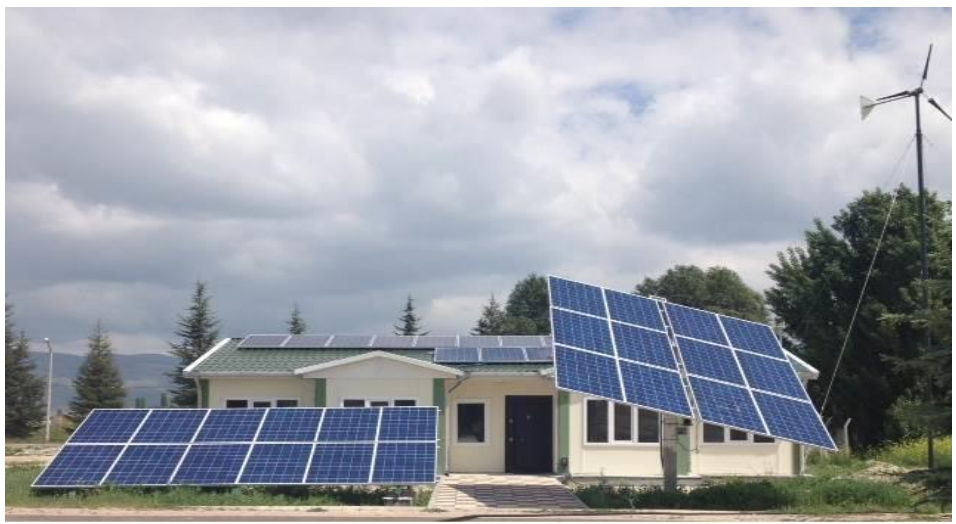

Fig. 1. (a) 12 panels $(260 \mathrm{~W})$ with one-axis (right) and without (left) solar tracker platform (b) averaged total daily power generation values of tracking and fixed panels.

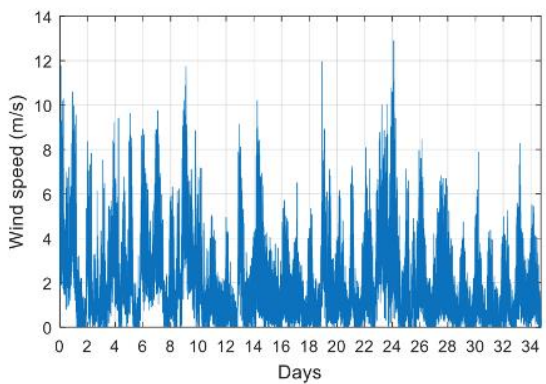

(a) Wind speed

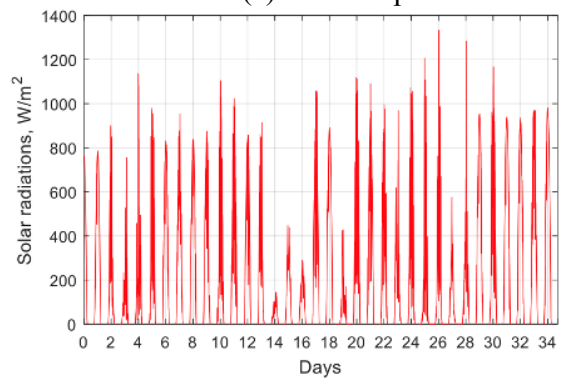

(c) Solar radiation

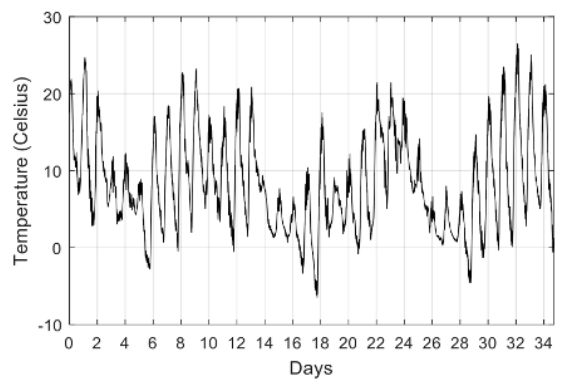

(b) Temperature

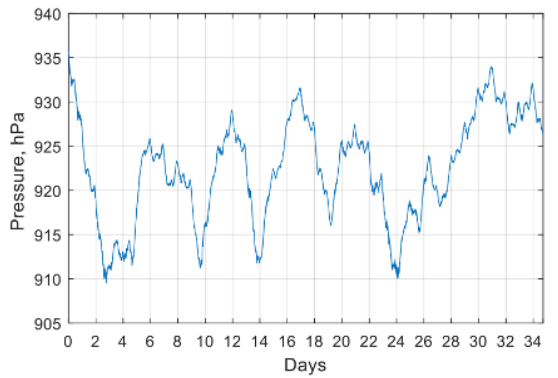

(d) Pressure

Fig. 2. Sample 20 seconds interval recoded (a) wind speed (b) temperature (c) solar radiation and (d) pressure values.

The electricity generation output of the active sun tracking and fixed PV panels are also recorded with 5 minutes' intervals as in Figure 3 (a) and (b), respectively. These figures use 
a compact $2 \mathrm{D}$ representation, where the produced electrical power is illustrated as a $\mathrm{z}$-axis value, and the $\mathrm{x}-\mathrm{y}$ axes consist of day-hour values. It can be seen that the power values have similar values along the same hour of consecutive days. Solar-wise, a highly varying season of March is selected here for the illustration of a relatively tough example. The geo location of the solar panels is $(39.81 \mathrm{E}, 30.54 \mathrm{~N})$, corresponding to an inland region in Anatolia. Similar observations are expected to be observed in around inland geographical regions with latitude around 28-33.
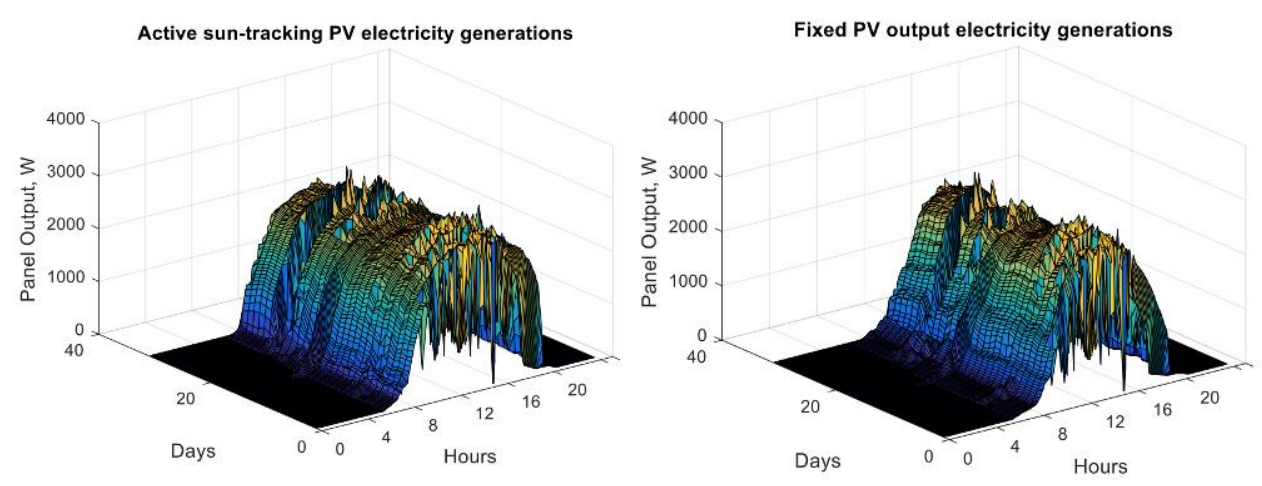

Fig. 3. The recorded real electricity generation output of the active sun tracking (left) and fixed PV panels (right) during March 2016 in Eskişehir region @ (39.81 E, 30.54 N).

The final data of interest for sizing applications are the actual prices and installation costs of the units. A list of unit costs for the experimental set-up items is given in Table 1. These values are per-unit scaled versions of real system components. Since the total cost of the inverter and battery charging regulator is directly proportional to the component costs they are not included in the calculations.

Table 1. Unit costs of ready-to-serve commercial products within Figure 1.

\begin{tabular}{|c|c|}
\hline Component & Cost (\$) \\
\hline Fixed PV panel (260 W) & 160 \\
\hline Active-sun track PV panel (260 W) & 312 \\
\hline Wind Tribune (260 W)-scaled & 500 \\
\hline 12 V, 200 Ah Battery & 350 \\
\hline
\end{tabular}

\subsection{Data modelling}

In this part, we propose a method to extend the recorded data to various scenarios in order to better assess the sizing result. The proposed extension method helps us to observe more accurate average performance of the sizing, as well as situations corresponding to worst case scenarios. It is claimed that the described data generation models can be extended for 
different geographical locations using by simply changing the defined parameters (such as mean/variance functions). Then, a sizing procedure can be simulated and compared for more stable results. In general, stochastic parameters are estimated from data, and applied to a random process generator that exhibits data for the extension purposes [7].

\subsubsection{Wind data}

A sample portion of the collected 20-seconds-interval wind speed values (corresponding to March) is plotted in Figure 4 (a). Each day (during March) is represented with a different color, and 24-hour data are plotted on top of each other. The average of the days along the hour axis is shown as solid black. Since the acquisition resolution is very high, timewindowed mean and variance values also exhibit a continuous variation (Figure 4 (b)).

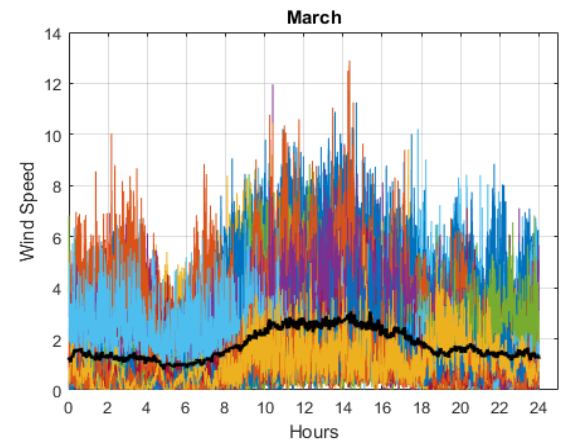

(a) The collected wind speed values for March.
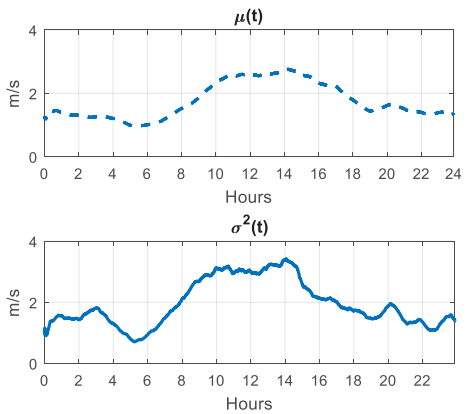

(b) Mean and variance functions.

Fig. 4. Sample wind speed plots.

Again, due to the high resolution the effective randomness of the wind speed data the time averages fit into locally normal or chi-squared distributions, while the distribution parameters (mean and variance) slowly change in time. This time varying distribution can be expressed according to the formula in Eq. 1.

$$
w_{i}(t)=\mathrm{N}\left(\mu_{i}(t), \sigma_{i}^{2}(t)\right)
$$

where $\mu_{i}(t)$ and $\sigma^{2}{ }_{i}(t)$ are timely varying mean and variance functions of the $i^{\text {th }}$ month. Therefore, the model slowly varies with the month index, $i$, and also varies in time, $t$.

For the modelling of solar radiation and the corresponding electrical energy, atmospheric conditions are also considered. However, a rougher set of values are used for modelling the atmospheric conditions: open, partly or fully cloudy, as explained in the next subsection.

\subsubsection{Solar radiation and power output models for active sun tracking PV}

Both the solar radiation and the corresponding electrical power depend on the extraterrestrial radiation model as well as the atmospheric conditions in the PV location at the given date. Figure 5 shows three example recorded electricity generation value plots of single-axis active sun tracking (blue curves) and fixed PV panels (red curves) for sample sunny, partly cloudy and cloudy days. They all have different characteristics. Since all the 
efficiencies and electricity generation characteristics are changing according to these three situations we propose a state varying procedure. An expected-but-important observation is that the blue curves corresponding to the sun tracking PV is consistently outperforming the analogous fixed panel energy production values.
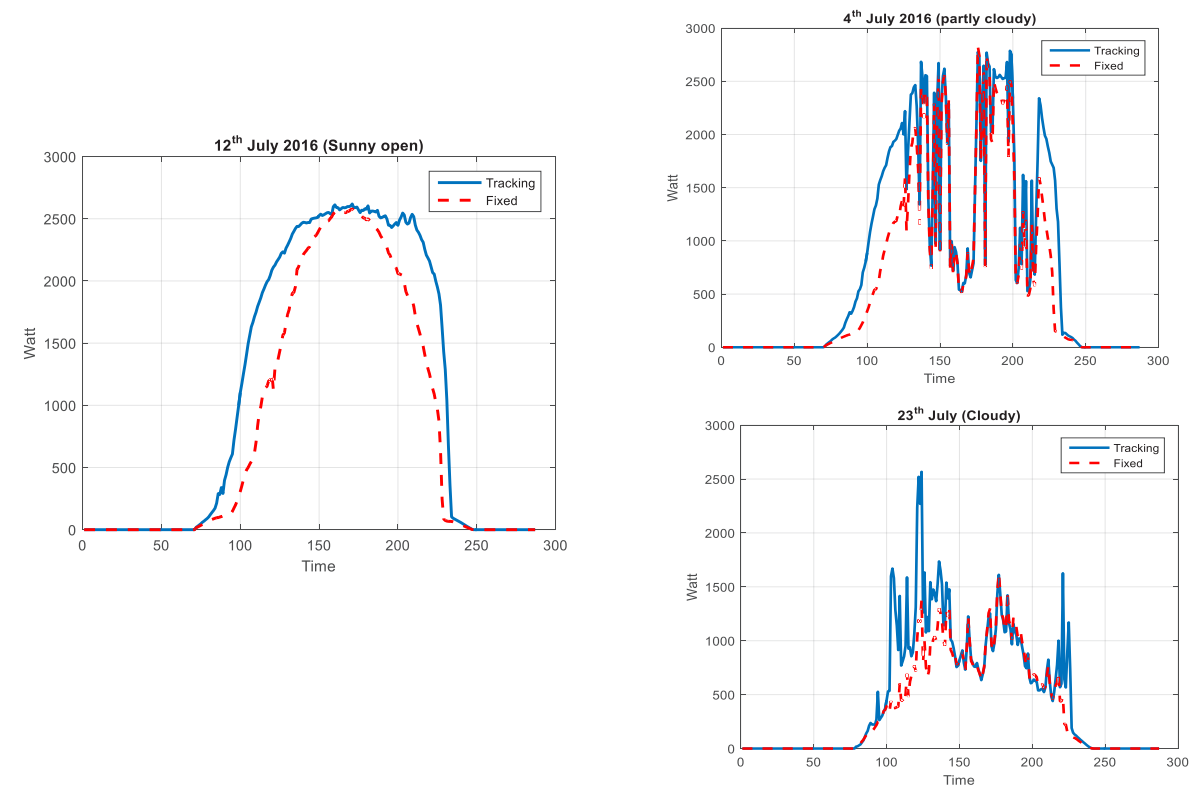

Fig. 5. Sample active sun tracking and fixed PV panel output electricity generations of sunny, partly cloudy, cloudy days.

The proposed state-varying atmospheric model can be illustrated with a 3 -state chain as illustrated in Figure 6. First, for each month all the state probabilities are calculated according to the total number of sunny, partly cloudy and cloudy days. Then state transitions are counted to obtain transition probabilities.

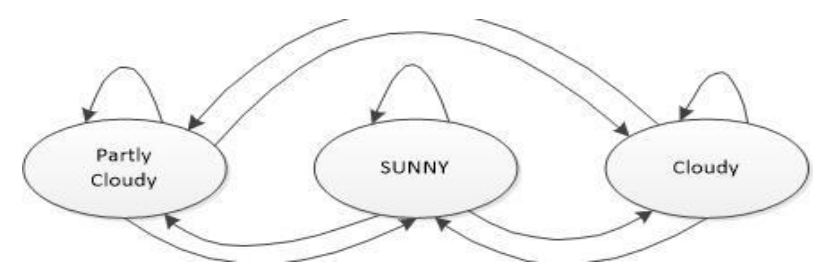

Fig. 6. Three atmospheric condition states with transition routes illustrated as arrows.

For a sunny day solar radiation values are modelled as truncated normal random process with calculated mean and variance functions as:

$$
g_{i, s}(t)=\mathrm{N}\left(\mu_{i, \text { sunnv }}(t), \sigma^{2}{ }_{i}(t)\right)
$$

For a partly cloudy day the cloud arrival probability is calculated with Poisson point process which is notated as $\mathrm{P}\left(\lambda_{i}(t)\right)$. With this model, solar radiation value can be generated by switching between the mean function of sunny and cloudy days' mean 
functions. Poisson process will decide the switch instants of the mean functions according to the distributions:

$$
\begin{gathered}
g_{i, p c}(t)=\mathrm{N}\left(\left(\mu_{i, \text { cloudy }}(t),\left(\mu_{i, \text { sunn } y}(t)\right), \sigma^{2}{ }_{i}(t)\right)\right. \\
g_{i, \text { cloudy }}(t)=\mathrm{N}\left(\mu_{i, \text { cloudy }}(t), \sigma^{2}{ }_{i}(t)\right)
\end{gathered}
$$

Once an extended set of data is generated from the above model, The sizing result that is obtained from the actual data can be tested for average and worst case scenario analysis.

\subsubsection{Electricity demand model}

Depending on the season, the electricity demand varies in the household. The yearly average electricity demand is measured as $6.38 \mathrm{kWh}$. Figure 7 depicts three sample daily demand profiles.
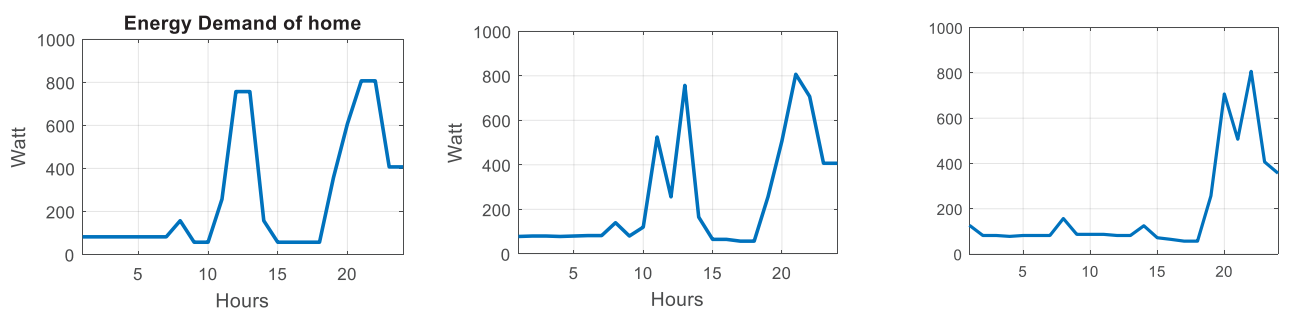

Fig. 7. Sample electric power demand profiles for three separate days in different seasons.

\section{Sizing procedure}

The actual recorded data are used for sizing the system to a minimal cost situation. Then, the achieved size is tested over the model simulations for improved assessment. During the sizing process, a new and efficient procedure is adopted [3]. The procedure uses all available renewable energy sources and starts by assuming maximum battery size (yielding a capacity to store all excess electric power and dispatches the storage whenever necessary). Then, gradually, the battery size is reduced while monitoring the LLP costs. On an axis of battery size, the LLP cost curve forms a decreasing plot. However, the batteries themselves contribute to the cost, producing a linearly increasing curve on the same axis. The combination of these two costs form the total cost, which exhibits a minimum value as illustrated in Figure 8. This optimal point is, then, tested against model generated data.

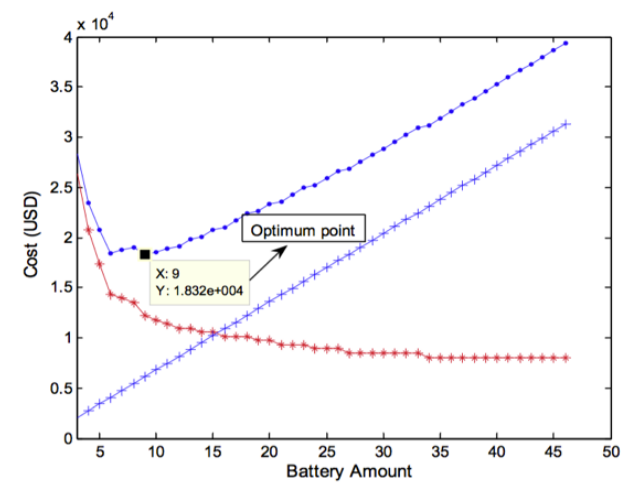

Fig. 8. Costs for LLP (red) and battery (blue cross) adding up to the total cost (blue dotted). 


\section{Results and discussions}

Using the recorded data, the system which contains fixed PV panels (260 W, \$160) and the wind turbines $(260 \mathrm{~W}, \$ 500)$ provided a minimum cost point at the battery size of 4 , corresponding to an LLP level of below 0.0100 and a total cost of $160+500+4 \times 350=\$ 2060$. When this system was tested for the model generated data, the LLP cost was observed to have an average value of 0.0092 with a worst case value of 0.0120. Then, the system is considered to have the sun tracking PV panels (260 W, \$312) together with the same wind turbines. As expected, the sizing produced a minimum cost at a point with less batteries ( 3 of them) at the same LLP levels. Although the initial cost of this version is higher than the one with fixed panels, the total cost is still lower $(312+500+3 \times 350=\$ 1862)$. Besides, the larger values achieved by the tracking system (blue curves in Figure 5) pays off well to perform better on the worst case scenarios with an LLP of 0.0092 on the average and 0.0109 at the worst case. If the building performs critical electricity-dependent operations, or if the life cycle assessment of the electrical products is considered, the worst case improvement of 0.0109 versus 0.0120 might be even more critical than the total investment cost optimization of $\$ 1862$ versus $\$ 2060$, which is rather marginal. It is concluded that utilization of a sun tracker PV panels is more efficient as it demands less battery storage at the same or better LLP levels. The situation is expected to be valid in similar geographical locations (i.e. inland regions with latitude 28-33), but such a conclusion requires more experimental works and verifications throughout the world.

\section{References}

1. W. Zhou, C. Lou, Z. Li, L. Lu, H. Yang, Applied Energy 87, 2, 380-389 (2010)

2. H. Yang, L. Lu, W. Zhou, Solar Energy 81, 1, 76-84 (2007)

3. F.O. Hocaoğlu, O.N. Gerek, M. Kurban, Solar Energy 83, 11, 2019-2028 (2009)

4. T. Filik, U.B. Filik, Anadolu U. J. of Sci. \& Tech.-A: App. Sci. \& Eng. (to appear, 2017)

5. S. Diaf, D. Diaf, M. Belhamel, M.A. Haddadi, A. Louche, Energy Policy 35, 5708-5718 (2017)

6. A.R. Prasad, E. Natarajan, Energy 31, 1943-1954 (2006)

7. R.Y. Rubinstein, D.P. Kroese, Simulation and the Monte Carlo Method (3.E.) (Wiley, 2017)

8. N. Maisonneuve, G. Gross, IEEE Trans. Power Sys. 26, 4 (2011)

9. A. Naimo, Procedia - Soc. \& Beh. Sci. 108, 187-196 (2014)

10. T. Kahlib, A. Mohamed, K. Sopiah, Ren. \& Sust. Energy Rev. 16, 5 (2012)

11. F.O. Hocaoğlu, O.N. Gerek, M. Kurban, Solar Energy 82, 8 (2008) 